\title{
Clinicopathological Significance of Tumor Stem Cell Markers ALDH1 and CD133 in Colorectal Carcinoma
}

\author{
Maryam Rezaee $^{1+(i D}$, Elmira Gheytanchi $^{1 \#}$, Zahra Madjd $^{1,2}$, Mitra Mehrazma $^{1,3 *}$ \\ 1. Oncopathology Research Center, Iran University of Medical Sciences, Tehran, Iran \\ 2. Department of Molecular Medicine, Faculty of Advanced Technologies in Medicine, Iran University of Medical Sciences, Tehran, Iran \\ 3. Department of Pathology, Hasheminejad Kidney Center, Iran University of Medical Sciences, Tehran, Iran
}

\begin{tabular}{c}
\hline KEYWORDS \\
ALDH1, \\
CDncer Stem Cells, \\
CD133, \\
Colorectal cancer \\
Scan to discover online \\
Q \\
GI, Liver \& Pancreas Pathology \\
Received 11 May 2020; \\
Accepted 23 Sep 2020; \\
Published Online 10 Oct 2020; \\
\hline
\end{tabular}

10.30699/ijp.2020.127441.2389

\section{ABSTRACT}

Background \& Objective: Colorectal cancer (CRC) is the third most common cancer worldwide with a high mortality rate. The main causes of death in patients are recurrence and metastasis which are mainly attributed to the small subpopulation of cells within tumors called cancer stem cells (CSCs). This study aimed to evaluate the correlation between the expression of ALDH1 and CD133 as CSC associated markers and clinicopathological characteristics in CRC.

Methods: In this cross-sectional study, a total of 483 CRC tumor samples were immunohistochemically stained for detection of CD133 and ALDH1 markers. Correlations of marker expression with clinicopathological factors were also evaluated.

Results: There was a significant correlation between the luminal intensity of CD133 and neural invasion $(P=0.05)$ and between the cytoplasmic intensity of CD133 and metastasis $(P=0.05)$. In terms of $\mathrm{H}$-score, a positive significant relation was observed between cytoplasmic expression of CD133 and lymph node $(P=0.02)$, neural $(P=0.04)$ and vascular invasion $(P=0.02)$. The ALDH1 cytoplasmic expression showed a significant correlation with tumor size $(P=0.001)$.

Conclusion: Our findings showed that increased expression of CD133 and ALDH1 is associated with tumor progression and worse outcomes in CRC patients. These markers can be good candidates for localized targeting of CSCs using antibodies. Future researches need to be improved approaches for early detection of CRC, and treatment monitoring for CRC and other cancers.

Mitra Mehrazma, Department of Pathology, Hasheminejad Kidney Center, Iran University of Medical Sciences, Tehran, Iran. E-mail: mitmehr@yahoo.com

\# Maryam Rezaee and Elmira Gheytanchi contributed equally to this manuscript as co-first authors.

Copyright (C) 2021. This is an open-access article distributed under the terms of the Creative Commons Attribution- 4.0 International License which permits Share, copy and redistribution of the material in any medium or format or adapt, remix, transform, and build upon the material for any purpose, even commercially.

\section{Introduction}

Colorectal cancer is one of the third most prevalent cancers and the fourth cause of cancer-related mortality worldwide with 700,000 deaths annually (1-4). In terms of gender, CRC is considered as the second most common cancer in females and the third in males (5). The risk factors are related to habits or personal characteristics including age, history of chronic disease and lifestyle which are associated with CRC progression (3). Different mutations targeting genes such as tumor suppressors, DNA repairing, and oncogenes lead to CRC (6). Based on the gene mutation site, CRC could be categorized as sporadic (70\%); inherited (5\%) and familial (25\%) (7). The chromosomal instability (CIN), microsatellite instability (MSI) and CpG island methylator phenotype (CIMP) has been reported as being the main mechanisms involved in CRC pathogenesis (8). The main pathways such as WNT, TP53, MAPK/PI3K, TGF- $\beta$, and mutated genes of PTEN,
PIK3CA, C-MYC, SMAD2, SMAD4, BRAF and KRAS are affected by chromosomal changes and translocations (912). Despite the improvement in CRC diagnosis and therapy, the survival rate of patients with $\mathrm{CRC}$ still remains poor because of its drug resistance, metastasis, and recurrence $(13,14)$. Therefore, it is essential to develop and implement precise and suitable biomarkers to enhance diagnostic processes aiding clinicians in the detection of $\mathrm{CRC}$ in the earliest stages. Emerging evidence has shown that a subpopulation of cells also called tumor initiating or cancer stem cells (CSCs) with multi-potency and selfrenewal characteristics have a critical role in CRC pathophysiology (15-17). They display chemotherapy resistance, differentiation potential, and high tumorigenicity and could be a promising therapeutic approach for CRC management $(18,19)$. CSCs has been effectively recognized in different solid tumors including 
CRC (20). Targeting of CSCs could be achieved in CRC through the various cell surface markers associated with self-renewal, including CD133, CD166, CD44, CD24, beta1 integrin-CD29, Lgr5, EpCAM (ESA), ALDH-1, Msi-1, DCAMLK1 or EphB receptors $(18,21)$. Hence, understanding which of these markers has the greatest effect on a patient's diagnosis and prognosis has been the focus of many studies. The CD133 and ALDH-1 are among the main markers that have been linked to CSCs in CRC (22).

The cell surface marker of CD133 also known as $\mathrm{AC} 133$ in humans or prominin-1 in rodents is a five domain transmembrane molecule with a molecular weight of 120 $\mathrm{kDa}$ that has been identified as a normal and putative CSC marker in several cancers, including brain tumors, prostate carcinoma and CRC (23-29). Previous studies reported the in-vitro and in-vivo self-renewal and high tumorigenicity potential of CD133 in CRC $(28,30)$. It has been demonstrated that the CD133 expression in combination with other putative CSC markers correlated with clinical outcomes in CRC patients (31).

Aldehyde dehydrogenase (ALDH) with several isoforms and different cellular functions and tissue distribution is located on chromosome $12(30,32)$. It is considered as a detoxifying enzyme for oxidation of intracellular aldehydes $(32,33)$. Isoforms of ALDH including ALDH-1 may lead to poor prognosis in patients and have been reported to be a putative stem cell marker in several cancers such as breast cancer (34), pancreatic adenocarcinoma (35), ovarian cancer (32) lung cancer and colorectal cancer $(30,32,36)$. Resistance to chemotherapy is attributed to the transcriptional triggering in ALDH1 leading to drug and radiation resistance in CSCs which was first detected in hematopoietic progenitor cells (32). The role of ALDH-1 and its isoforms in CSCs characteristics including self-renewal, differentiation and epithelialmesenchymal transition (EMT) has also been shown in xenotransplants of breast cancer and colon cancer $(32,37$, 38). Previous studies in the literature indicated the prognostic value of ALDH1 expression in different cancers including CRC $(39,40)$ and some of them showed that there was any relation between the ALDH1 expression and tumor stage (41).

Considering the lack of comprehensive information focusing on the relationship between concurrent expression of putative CSC markers ALDH1 and CD133 with main clinicopathological factors in CRC, current study was aimed to evaluate the immunohistochemical expression of these markers in CRC patients.

\section{Materials and Methods}

\section{Patient's Data Collection and Sample Preparation}

In this cross-sectional study, formalin- fixed-paraffinembedded (FFPE) tissues from 483 patients, who were diagnosed with $\mathrm{CRC}$, were collected from three University-based Referral Centers of Hasheminejad, Rasoul-Akram and Firoozgar in Tehran, Iran, from 2009 to 2015. Patients' medical records were evaluated to ensure the following demographic and clinicopathological parameters were included, those being, age, gender, tumor size, grade, and stage, tumor location, tumor differentiation status, metastasis, lymph node and neural tissue invasion. All tumor histology was assessed using the hematoxylin and eosin (H\&E) stained slides of tumors by experienced pathologists. Furthermore, patient data was kept confidential and the ethical use of patients'tissue samples was approved by the Ethics Committee of the Iran University of Medical Sciences (IR.IUMS.REC 1395.9311100010).

\section{Construction of Tissue Microarray (TMA) Blocks}

The CRC TMA blocks were prepared as aforementioned (40). In brief, the selection of tumor representative areas was performed from $\mathrm{H} \& \mathrm{E}$ slides for TMA preparation. The cores with a $0.6 \mathrm{~mm}$ diameter from marked tumor areas were punched into a tissue microarray block by Tissue Arrayer Minicore (ALPHELYS, Plaisir, France). The TMAs were constructed in five copies for each sample, the mean scoring of cores was then considered as the final score (27). Finally, the sectioning of TMA blocks was performed for immunohistochemical staining.

\section{Immunohistochemical (IHC) Determination of Markers}

The expression level of ALDH1 and CD133 was evaluated in CRC sections by the IHC method, as described previously (30). In sum, paraffin-embedded human CRC TMA blocks were sectioned at 5- $\mu \mathrm{m}$ thickness and then mounted onto Super frost slides (Superfrost plus, Thermo Scientific, Germany). All slides were dewaxed at $60^{\circ} \mathrm{C}$ for $30 \mathrm{~min}$, deparaffinized with xylene and rehydrated in ethylic alcohol serial dilution and then treated with 3\% hydrogen peroxide for $20 \mathrm{~min}$. For antigenic epitope demasking the antigen retrieval process was done using pressure-cooking system by submerging in citrate buffer ( $\mathrm{pH}: 6.0$ ) as target retrieval solution of both markers. After cooling the slides at room temperature, they were rinsed in Tris-buffered saline (TBS) three times. For primary staining of both markers, slides were respectively incubated with rabbit recombinant monoclonal ALDH1A1 antibody (1:200, overnight at $40^{\circ} \mathrm{C}$, ab52492; Abcam; USA) and rabbit polyclonal CD133 antibody $\left(1: 250\right.$, overnight at $40^{\circ} \mathrm{C}$, ab19898; Abcam; USA). Secondary staining was performed using EnVision TM/HRP, Rabbit/Mouse reagent (Dako; code K5007; Denmark; Ready to-use) for $30 \mathrm{~min}$ in a wet box at $37^{\circ} \mathrm{C}$ and then visualized by Dako REALTM DAB + Chromogen (Dako; Denmark) based on the manufacturer's instructions. After washing in TBS, slides were finally counterstained with Mayer's hematoxylin dye (Dako; Denmark) for $15 \mathrm{~min}$, and dehydrated in ethylic alcohol serial dilution and cleared in xylene.

\section{Immunostaining Evaluation and Scoring System}

Imaging analysis of the CRC TMA cores for ALDH1 and CD133 expression was performed in a coded manner independently by two pathologists (M.M. and M.R.) without knowledge about the patients' clinicopathological characteristics. Discrepancies between them were resolved by consensus. The staining percentage of 
cytoplasmic and luminal expression of CD133 and ALDH1, respectively, scored as follows: 1 ; less than $25 \%$, score $2 ; 26-50 \%$, score $3 ; 51-75 \%$ and score 4 ; more than $75 \%$. The staining intensity was evaluated using a semiquantitative system, ranging from non-stained to strong: 0 ; non-staining, 1; weakly staining, 2; moderate staining, 3; strong staining. The histochemical score results were finally calculated by multiplying the intensity in total percentage of positive cells ranging from 0-300 including less than 100, between 100-200 and more than 200.

\section{Statistical Analysis}

All statistical analyses were performed using SPSS version 22 (SPSS Inc., Chicago, IL., USA). Differences in ALDH1 and CD133, based on different clinicopathological parameters, were estimated by a $\chi 2$ test or Fisher's exact test, if appropriate. The statistically significant difference was considered as $\mathrm{P}$-value $<0.05$ and all statistics are presented to two decimal places.

\section{Results Patients \\ Clinicopathological and Tumor Characteristics of}

In the present cross-sectional study, a total of 483 patients, diagnosed with colorectal adenocarcinoma, were included in this study. Out of 483 cases, $52.4 \%$ of cases were male and $47.6 \%$ of them were female with a mean age of 59.12 \pm 14.8 (range 23-92 years) at the time of diagnosis. Of all patients, $46.2 \%$ were $\geq 60$ years old and $53.8 \%$ were $<60$ years old. The pathological results showed that the most prevalent tumor location was sigmoid (164 cases, 35.5\%), and rectum (129 cases, $28 \%$ ). The mean size of tumors was $5 \mathrm{~cm}, 324(67.1 \%)$ tumors were less than $5 \mathrm{~cm}$ and 159 (32.9\%) were more than $5 \mathrm{~cm}$. Tumor cells were classified as well, moderately and poorly differentiated. Out of all tumor samples, there were 173 (35.8\%) well-differentiated, 277 $(57.3 \%)$ moderately differentiated and $33(6.8 \%)$ poorly differentiated adenocarcinoma cells. Thirty-four $(7 \%)$ patients showed metastasis and 449 (93\%) cases had no metastasis. Lymph node involvement was present in 181 patients $(37.5 \%)$ and absent in 302 patients $(62.5 \%)$. Neural invasion was observed in $97(20.1 \%)$ cases and non-invasion in $386(79.9 \%)$ cases and $74(15.3 \%)$ cases had vascular invasion. There were $84(17.4 \%)$ TNM stage 1, 208 (43.1\%) TNM stage 2, 168 (34.8\%) TNM stage 3 and $23(4.8 \%)$ TNM stage 4 patients. The highest prevalence of tumor stage was $2 \mathrm{a}(38 \%)$.

Correlation Between the Positive Expression of CD133 and CRC Clinicopathological Features

The CD133 was expressed in cytoplasm of 358 and luminal area of 315 tumor samples and intensity of staining in CRC tissues are shown in Figure 1. A number of tumor samples were excluded from the study due to the tissue handling process or lack of tumor within the cores. The expression of CD133 varied considerably between different tumor samples. In terms of expression intensity, strong luminal intensity was observed in $75.9 \%$ of samples, and $42.7 \%$ of cases showed strong cytoplasmic intensity.

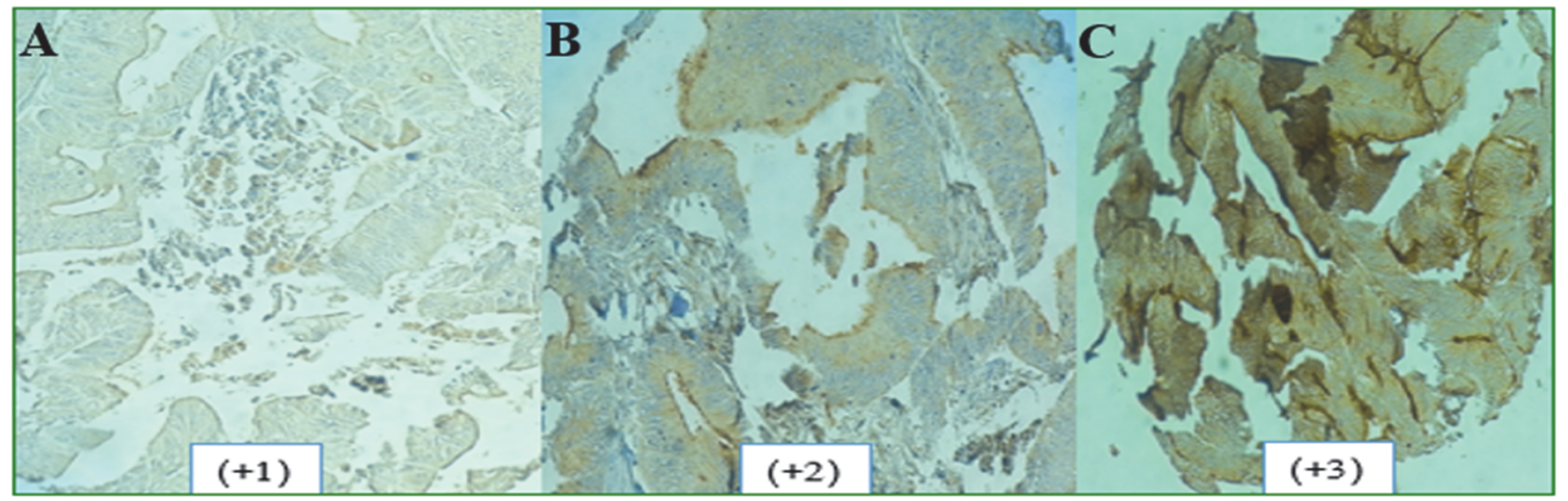

Fig. 1. Expression patterns of CD133 in CRC tissues. (A) Weak; (B) Moderate; and (C) Strong intensity of staining.

As indicated in Table 1, overall luminal and cytoplasmic expression of CD133 was not significantly associated with gender, age, tumor size $(P>0.05)$. 66.1\% of samples which showed neural invasion had high expression of CD133. There was significant correlation between the luminal intensity of CD133 expression and neural invasion $(P=0.05)$. High expression of CD133 was found in $79.4 \%$ of tumor samples with lymph node involvement. However, no significant correlation was identified between strong luminal and cytoplasmic expression of CD133 and lymph node involvement. 40\% of patients with metastasis showed high cytoplasmic expression of CD133 compared to $66.7 \%$ of metastatic cases with high luminal expression which was significant $(P=0.05)$. A higher CD133 luminal intensity was detected in $76.4 \%$ of moderately or poorly differentiated malignant tumor cells compared with the well $(75 \%)$ differentiated tumor cells. These values were relatively low in cytoplasmic expression of CD133. There was no significant association between luminal and cytoplasmic CD133 expression and tumor differentiation.

In terms of CD133 expression $\mathrm{H}$-score as indicated in Table $1,64.6 \%$ of male and $69.3 \%$ of female cases showed high H-score $(>200)$ of luminal CD133 expression compared to $33.2 \%$ male and $26.8 \%$ female 
in cytoplasmic expression of CD133. 64.7. \% of cases with H-score higher than 200 had tumor size more than $5 \mathrm{~cm}$ and $55 \%$ of tumors with vascular invasion had higher H-score $(>200)$ and $55.4 \%$ of tumors with neural invasion showed H-score higher than 200. The lymph nodes were involved in $73(68.2 \%)$ cases with higher Hscore. There was no significant association between the luminal and cytoplasmic H-score of CD133 with age, gender, tumor size, and metastasis $(\mathrm{P}>0.05)$. The luminal $\mathrm{H}$-score of CD133 presented marked correlation with neural invasion $(\mathrm{P}=0.04)$. There was a significant association between the cytoplasmic H-score of CD133 with clinical factors including lymph node $(P=0.02)$, neural $(P=0.04)$, and vascular $(P=0.02)$ invasion.

Table 1. Correlation between intensity and H-score of CD133 expression and clinicopathological characteristics in CRC.

\begin{tabular}{|c|c|c|c|c|c|c|c|c|}
\hline $\begin{array}{c}\text { Patients and } \\
\text { tumor } \\
\text { characteristics }\end{array}$ & $\begin{array}{l}\text { H-score } \\
\text { expressio }\end{array}$ & $\begin{array}{l}\text { CD133 } \\
\text { No. }(\%)\end{array}$ & P-value & & Intensity of & 3 expressior & & P-value \\
\hline $\begin{array}{l}\text { Expression } \\
\text { Pattern }\end{array}$ & Low & High & & Negative & Weak & Moderate & Strong & \\
\hline Cytoplasmic & $250(69.8)$ & $108(30.2)$ & & $3(0.8)$ & $77(21.5)$ & $125(34.9)$ & $153(42.7)$ & \\
\hline Luminal & $105(33.3)$ & $210(66.7)$ & & $8(2.5)$ & $25(7.9)$ & $43(13.7)$ & $239(75.9)$ & \\
\hline $\begin{array}{l}\text { Age } \\
\geq \mathbf{6 0} \\
\text { Cytoplasmic } \\
\text { Luminal } \\
<\mathbf{6 0} \\
\text { Cytoplasmic } \\
\text { Luminal }\end{array}$ & $\begin{array}{c}130(69.1) \\
55(32) \\
120(70.6) \\
50(35)\end{array}$ & $\begin{array}{c}58(30.9) \\
117(68) \\
50(29.4) \\
93(65)\end{array}$ & $\begin{array}{l}0.76 \\
0.57\end{array}$ & $\begin{array}{l}1(0.5) \\
4(2.3) \\
2(1.2) \\
4(2.8)\end{array}$ & $\begin{array}{c}39(20.7) \\
13(7.6) \\
38(22.4) \\
12(8.4)\end{array}$ & $\begin{array}{l}68(36.2) \\
21(12.2) \\
57(33.5) \\
22(15.4)\end{array}$ & $\begin{array}{c}80(42.6) \\
134(77.9) \\
73(42.9) \\
105(73.4)\end{array}$ & $\begin{array}{l}0.86 \\
0.82\end{array}$ \\
\hline $\begin{array}{l}\text { Gender } \\
\text { Male } \\
\text { Cytoplasmic } \\
\text { Luminal } \\
\text { Female } \\
\text { Cytoplasmic } \\
\text { Luminal }\end{array}$ & $\begin{array}{c}123(73.2) \\
43(30.7)\end{array}$ & $\begin{array}{c}63(33.2) \\
113(64.6) \\
45(26.8) \\
97(69.3)\end{array}$ & $\begin{array}{l}0.19 \\
0.37\end{array}$ & $\begin{array}{l}1(0.5) \\
5(2.9) \\
2(1.2) \\
3(2.1)\end{array}$ & $\begin{array}{c}44(23.2) \\
16(9.1) \\
33(19.6) \\
9(6.4)\end{array}$ & $\begin{array}{l}60(31.6) \\
26(14.9) \\
65(38.7) \\
17(12.1)\end{array}$ & $\begin{array}{c}85(44.7) \\
128(73.1) \\
68(40.5) \\
111(79.3)\end{array}$ & $\begin{array}{l}0.44 \\
0.64\end{array}$ \\
\hline $\begin{array}{l}\text { Tumor size } \\
\leq 5 \\
\text { Cytoplasmic } \\
\text { Luminal } \\
>5 \\
\text { Cytoplasmic } \\
\text { Luminal }\end{array}$ & $\begin{array}{c}164(68.3) \\
69(32.4) \\
86(72.9) \\
36(35.3)\end{array}$ & $\begin{array}{c}76(31.7) \\
144(67.6) \\
32(27.1) \\
66(64.7)\end{array}$ & $\begin{array}{l}0.60 \\
0.71\end{array}$ & $\begin{array}{l}1(0.4) \\
5(2.3) \\
2(1.7) \\
3(2.9)\end{array}$ & $\begin{array}{c}51(21.3) \\
17(8) \\
26(22) \\
8(7.8)\end{array}$ & $\begin{array}{l}82(34.2) \\
25(11.7) \\
43(36.4) \\
18(17.6)\end{array}$ & $\begin{array}{l}106(44.2) \\
166(77.9) \\
47(39.8) \\
73(71.6)\end{array}$ & $\begin{array}{l}0.56 \\
0.52\end{array}$ \\
\hline $\begin{array}{l}\text { Vascular } \\
\text { invasion } \\
\text { Yes } \\
\text { Cytoplasmic } \\
\text { Luminal } \\
\text { No } \\
\text { Cytoplasmic } \\
\text { Luminal }\end{array}$ & $\begin{array}{c}41(83.7) \\
18(45) \\
209(67.6) \\
87(31.6)\end{array}$ & $\begin{array}{c}8(16.3) \\
22(55) \\
100(32.4) \\
188(68.4)\end{array}$ & $\begin{array}{l}\mathbf{0 . 0 2} \\
0.09\end{array}$ & $\begin{array}{l}1(2) \\
2(5) \\
2(0.6) \\
6(2.2)\end{array}$ & $\begin{array}{c}14(28.6) \\
2(5) \\
63(20.4) \\
23(8.4)\end{array}$ & $\begin{array}{c}20(40.8) \\
8(20) \\
105(34) \\
35(12.7)\end{array}$ & $\begin{array}{c}14(28.6) \\
28(70) \\
139(45) \\
211(76.7)\end{array}$ & $\begin{array}{l}0.13 \\
0.36\end{array}$ \\
\hline $\begin{array}{l}\text { Neural invasion } \\
\text { Yes } \\
\text { Cytoplasmic } \\
\text { Luminal } \\
\text { No } \\
\text { Cytoplasmic } \\
\text { Luminal }\end{array}$ & $\begin{array}{c}190(57.4) \\
80(30.9)\end{array}$ & $\begin{array}{l}104(31.4) \\
179(69.1)\end{array}$ & $\begin{array}{l}0.04 \\
0.04\end{array}$ & $\begin{array}{l}2(0.7) \\
7(2.7)\end{array}$ & $\begin{array}{c}14(21.2) \\
4(7.1) \\
63(21.6) \\
21(8.1)\end{array}$ & $\begin{array}{l}31(47) \\
14(25) \\
94(32.2) \\
29(11.2)\end{array}$ & $\begin{array}{c}20(30.3) \\
37(66.1) \\
133(45.5) \\
202(78)\end{array}$ & $\begin{array}{l}0.08 \\
\mathbf{0 . 0 5}\end{array}$ \\
\hline $\begin{array}{l}\text { Lymph node } \\
\text { involvement } \\
\text { Yes } \\
\text { Cytoplasmic } \\
\text { Luminal }\end{array}$ & $\begin{array}{l}53(80.3) \\
34(31.8)\end{array}$ & $\begin{array}{l}13(19.7) \\
73(68.2)\end{array}$ & $\begin{array}{l}\mathbf{0 . 0 2} \\
0.67\end{array}$ & $\begin{array}{l}1(0.8) \\
3(2.8)\end{array}$ & $\begin{array}{c}31(23.8) \\
7(6.5)\end{array}$ & $\begin{array}{l}49(37.7) \\
12(11.2)\end{array}$ & $\begin{array}{l}49(37.7) \\
85(79.4)\end{array}$ & $\begin{array}{l}0.53 \\
0.70\end{array}$ \\
\hline
\end{tabular}




\begin{tabular}{|c|c|c|c|c|c|c|c|c|}
\hline $\begin{array}{c}\text { Patients and } \\
\text { tumor } \\
\text { characteristics }\end{array}$ & $\begin{array}{r}\text { H-score } \\
\text { expressio }\end{array}$ & $\begin{array}{l}\text { CD133 } \\
\text { No. (\%) }\end{array}$ & P-value & & Intensity of & 3 expressio & & P-value \\
\hline $\begin{array}{l}\text { No } \\
\text { Cytoplasmic } \\
\text { Luminal }\end{array}$ & $\begin{array}{c}197(67.5) \\
18(34.1)\end{array}$ & $\begin{array}{c}95(32.5) \\
137(65.9)\end{array}$ & & $\begin{array}{l}2(0.9) \\
5(2.4)\end{array}$ & $\begin{array}{c}46(20.2) \\
18(8.7)\end{array}$ & $\begin{array}{l}76(33.3) \\
31(14.9)\end{array}$ & $\begin{array}{c}104(45.6) \\
154(74)\end{array}$ & \\
\hline $\begin{array}{l}\text { Metastasis } \\
\text { Yes } \\
\text { Cytoplasmic } \\
\text { Luminal } \\
\text { No } \\
\text { Cytoplasmic } \\
\text { Luminal }\end{array}$ & $\begin{array}{c}232(69.7) \\
96(32.7)\end{array}$ & $\begin{array}{c}7(28) \\
12(57.1) \\
101(30.3) \\
198(67.3)\end{array}$ & $\begin{array}{l}0.80 \\
0.33\end{array}$ & $\begin{array}{l}2(0.6) \\
7(2.4)\end{array}$ & $\begin{array}{c}9(36) \\
4(19) \\
68(20.4) \\
21(7.1)\end{array}$ & $\begin{array}{c}5(20) \\
2(9.5) \\
\\
120(36) \\
41(13.9)\end{array}$ & $\begin{array}{c}10(40) \\
14(66.7) \\
143(42.9) \\
225(76.5)\end{array}$ & $\begin{array}{l}\mathbf{0 . 0 5} \\
0.21\end{array}$ \\
\hline $\begin{array}{l}\text { Differentiation } \\
\text { Cytoplasmic } \\
\text { Well } \\
\text { Moderate \& Poor } \\
\text { Luminal } \\
\text { Well } \\
\text { Moderate \& Poor }\end{array}$ & $\begin{array}{l}44(36.7) \\
61(31.3)\end{array}$ & $\begin{array}{c}38(28.1) \\
70(31.4) \\
76(63.3) \\
134(68.7)\end{array}$ & 0.32 & $\begin{array}{l}1(0.8) \\
7(3.6)\end{array}$ & $\begin{array}{c}14(11.7) \\
11(5.6)\end{array}$ & $\begin{array}{l}15(12.5) \\
28(14.4)\end{array}$ & $\begin{array}{c}61(45.2) \\
92(41.3) \\
90(75) \\
149(76.4)\end{array}$ & 0.51 \\
\hline $\begin{array}{l}\text { Stage } \\
\text { Cytoplasmic } \\
\text { I \& II } \\
\text { III \& IV } \\
\text { Luminal } \\
\text { I \& II } \\
\text { III \& IV }\end{array}$ & $\begin{array}{l}69(34.3) \\
36(31.6)\end{array}$ & $\begin{array}{c}71(32.4) \\
37(26.6) \\
132(60.7) \\
78(68.4)\end{array}$ & 0.61 & $\begin{array}{c}2(0.9) \\
1(0.7) \\
\\
6(3) \\
2(1.8)\end{array}$ & $\begin{array}{c}46(21) \\
31(22.3)\end{array}$ & $\begin{array}{l}28(13.9) \\
15(13.2)\end{array}$ & $\begin{array}{c}100(45.7) \\
53(38.1) \\
149(74.1) \\
90(78.9)\end{array}$ & 0.70 \\
\hline
\end{tabular}

*The values are shown in bold are statistically significant.

Correlation Between the Positive Expression of ALDH1 and CRC Clinicopathological Features

The expression pattern of ALDH1 was mainly cytoplasmic in 40\% (167/416) of TMA tumors (Figure 2), whereas $60 \%$ of cases showed negative intensity of staining. High ALDH1 expression was distinguished in $4(2.7 \%)$ of the well-differentiated tumors, $3(1.3 \%)$ of moderately differentiated tumors and none of the highly expressed tumors showed poorly differentiated pattern. Seven cases with metastasis showed high ALDH1 expression. Only $1.3 \%, 2.1 \%$ and $1.7 \%$ of tumors with high intensity of staining developed lymph nodes, neural and vascular invasion, respectively (Table 2). The ALDH1 expression (intensity and $\mathrm{H}$-score) was not significantly associated with age, gender, lymph nodes, and vascular invasion $(P>0.05)$. Tumors more than $5 \mathrm{~cm}$ in size exhibited H-score higher than 200 in $22.5 \%$ of cases (Table 2). A considerable positive correlation was found between the tumor size and H-score of ALDH1 expression in tumor cells $(P=0.001)$. A marginal trend correlation was also observed between the neural invasion and ALDH1 intensity of staining $(P=0.07)$.

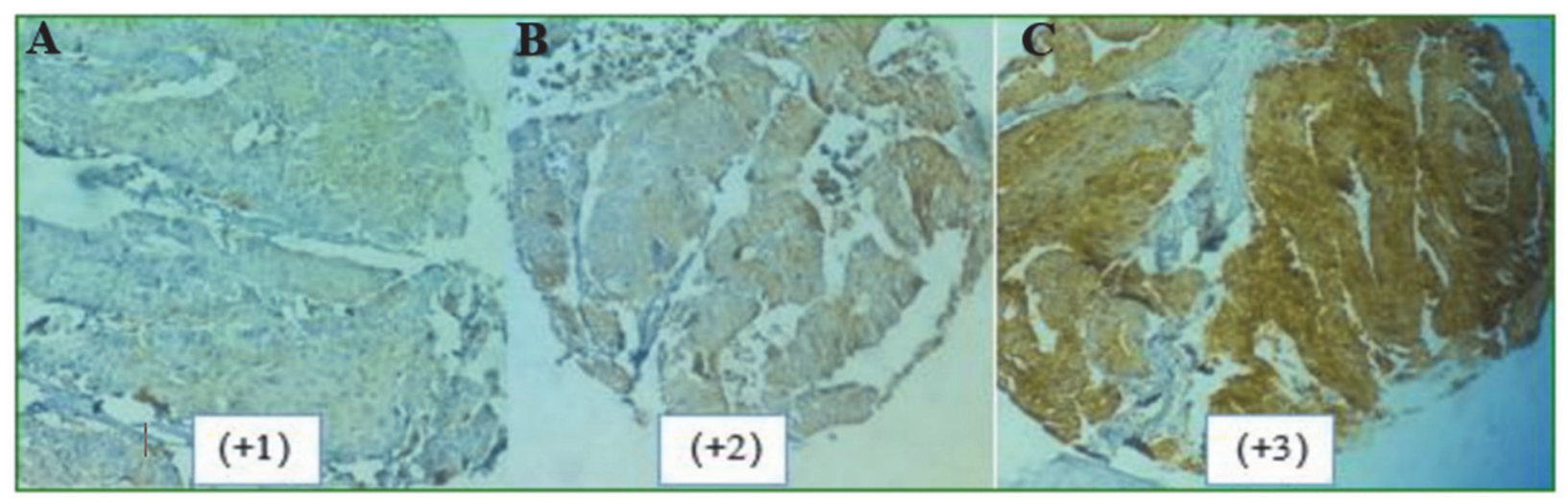

Fig. 2. Expression patterns of ALDH1 in CRC tissues. (A) Weak (B) Moderate; and (C) Strong intensity of staining. 
Table 2. Correlation between intensity and H-score of ALDH1 expression and clinicopathological characteristics in CRC.

\begin{tabular}{|c|c|c|c|c|c|c|c|c|c|}
\hline $\begin{array}{l}\text { Patients and } \\
\text { tumor }\end{array}$ & H-score of & H1 expre & No. (\%) & $P$ & Inte & y of ALDH & pression No & & $P$ \\
\hline $\begin{array}{l}\text { Expression } \\
\text { Pattern }\end{array}$ & $<100$ & $100-200$ & $<\mathbf{2 0 0}$ & & Negative & Weak & Moderate & Strong & \\
\hline Cytoplasmic & $405(96.9)$ & $11(2.6)$ & $2(0.5)$ & & $249(60)$ & $126(30)$ & $34(8)$ & $7(2)$ & \\
\hline $\begin{array}{l}\text { Gender } \\
\text { Male } \\
\text { Cytoplasmic } \\
\underline{\text { Female }} \\
\text { Cytoplasmic }\end{array}$ & $\begin{array}{l}207(95.4) \\
196(98.5)\end{array}$ & $\begin{array}{l}9(4.1) \\
2(1)\end{array}$ & $\begin{array}{l}1(0.5) \\
1(0.5)\end{array}$ & 0.09 & $\begin{array}{l}126(58) \\
123(61)\end{array}$ & $\begin{array}{l}69(32) \\
57(29)\end{array}$ & $\begin{array}{l}17(8) \\
17(8)\end{array}$ & $\begin{array}{l}4(2) \\
3(2)\end{array}$ & 0.88 \\
\hline $\begin{array}{l}\text { Age } \\
\underline{\mathbf{2 3 - 4 0}} \\
\text { Cytoplasmic } \\
\underline{\mathbf{4 1 - 6 0}} \\
\text { Cytoplasmic } \\
\underline{\mathbf{6 0}} \\
\text { Cytoplasmic }\end{array}$ & $170(98.3)$ & $\begin{array}{l}2(4.8) \\
3(1.7) \\
6(3)\end{array}$ & $\begin{array}{l}0(0) \\
2(1)\end{array}$ & 0.44 & $\begin{array}{l}100(57.8) \\
130(64.7)\end{array}$ & $\begin{array}{l}17(40.5) \\
56(32.4) \\
53(26.4)\end{array}$ & $\begin{array}{l}4(9.5) \\
16(9.2) \\
14(7)\end{array}$ & $\begin{array}{l}2(4.8) \\
1(0.6) \\
4(2)\end{array}$ & 0.11 \\
\hline $\begin{array}{l}\text { Tumor size } \\
\leq \underline{\mathbf{5}} \\
\text { Cytoplasmic } \\
\mathbf{2 5} \\
\text { Cytoplasmic }\end{array}$ & $\begin{array}{l}276(95.8) \\
129(93.3)\end{array}$ & $\begin{array}{l}2(3.4) \\
9(5.9)\end{array}$ & $\begin{array}{l}1(0.8) \\
1(0.8)\end{array}$ & 0.001 & $\begin{array}{l}163(59.4) \\
82(60.7)\end{array}$ & $\begin{array}{r}88(32) \\
37(27.4)\end{array}$ & $\begin{array}{l}20(7.3) \\
13(9.6)\end{array}$ & $\begin{array}{l}4(1.5) \\
3(2.2)\end{array}$ & 0.66 \\
\hline $\begin{array}{l}\text { Vascular } \\
\text { invasion } \\
\text { Yes } \\
\text { Cytoplasmic } \\
\underline{\text { No }} \\
\text { Cytoplasmic }\end{array}$ & $56(96.6)$ & 2(3.4) & $0(0)$ & 0.75 & $\begin{array}{l}36(62.1) \\
207(59.5)\end{array}$ & $\begin{array}{l}15(25.9) \\
107(30.7)\end{array}$ & $\begin{array}{l}6(10.3) \\
28(8)\end{array}$ & $\begin{array}{l}1(1.7) \\
6(1.7)\end{array}$ & 0.85 \\
\hline $\begin{array}{l}\text { Neural invasion } \\
\text { Yes } \\
\text { Cytoplasmic } \\
\underline{\text { No }} \\
\text { Cytoplasmic }\end{array}$ & $\begin{array}{l}79(98.6) \\
326(96.4)\end{array}$ & $\begin{array}{l}1(1.4) \\
10(3)\end{array}$ & $\begin{array}{r}0(0) \\
2(0.6)\end{array}$ & 0.79 & $\begin{array}{l}53(72.6) \\
190(57.4)\end{array}$ & $\begin{array}{l}17(23.3) \\
104(31.4)\end{array}$ & $\begin{array}{l}3(44.1) \\
30(9.1)\end{array}$ & $\begin{array}{c}0(0) \\
7(2.1)\end{array}$ & 0.07 \\
\hline $\begin{array}{l}\text { Lymph node } \\
\text { involvement } \\
\text { Yes } \\
\text { Cytoplasmic } \\
\text { No } \\
\text { Cytoplasmic }\end{array}$ & $82(97.1)$ & $3(2.1)$ & $\begin{array}{l}1(0.8) \\
1(0.8)\end{array}$ & 0.88 & $\begin{array}{l}82(54.3) \\
166(26.9)\end{array}$ & $\begin{array}{l}53(35.1) \\
73(27.7)\end{array}$ & $\begin{array}{l}14(9.3) \\
20(7.6)\end{array}$ & $\begin{array}{l}2(1.3) \\
5(1.9)\end{array}$ & 0.32 \\
\hline $\begin{array}{l}\text { Metastasis } \\
\underline{\text { Yes }} \\
\text { Cytoplasmic } \\
\underline{\text { No }} \\
\text { Cytoplasmic }\end{array}$ & $\begin{array}{l}31(95.8) \\
374(96.8)\end{array}$ & $\begin{array}{l}1(4.2) \\
10(2.6)\end{array}$ & $\begin{array}{l}0(0) \\
2(0.5)\end{array}$ & 0.55 & $\begin{array}{c}16(66.7) \\
225(59.8)\end{array}$ & $\begin{array}{c}7(29.2) \\
122(29.8)\end{array}$ & $\begin{array}{l}1(4.2) \\
32(8.5)\end{array}$ & $\begin{array}{c}0(0) \\
7(1.9)\end{array}$ & 0.93 \\
\hline $\begin{array}{l}\text { Differentiation } \\
\text { Cytoplasmic } \\
\text { Well } \\
\text { Moderate } \\
\text { Poor }\end{array}$ & $\begin{array}{c}140(94.6) \\
237(97.9) \\
28(100)\end{array}$ & $\begin{array}{c}7(4.8) \\
4(1.7) \\
0(0)\end{array}$ & $\begin{array}{c}1(0.7) \\
1(0.4) \\
0(0)\end{array}$ & 0.33 & $\begin{array}{c}82(56.2) \\
145(61.2) \\
18(66.7)\end{array}$ & $\begin{array}{c}45(30.8) \\
73(30.8) \\
6(22.2)\end{array}$ & $\begin{array}{c}15(10.3) \\
16(6.8) \\
3(11.1)\end{array}$ & $\begin{array}{c}4(2.7) \\
3(1.3) \\
0(0)\end{array}$ & 0.61 \\
\hline
\end{tabular}

*The values are shown in bold are statistically significant.

Correlation Between the Expression of CD133/ALDH1 and CRC Clinicopathological Features

The association between immunohistochemical CD133 and ALDH1 expression was measured in the
283 matched cases using Pearson's chi-square. A significant reciprocal relation was found between the CD133 and ALDH1 expression $(P<0.001)$. Based on the combined analysis, the CD133/ALDH1 expression was divided into 4 phenotypes, including CD133- 
High/ALDH1-High (8.8\%) indicating high expression of both markers, CD133-Low/ALDH1-Low (8.8\%) indicating low expression of both markers.CD133High/ALDH1-Low (81.3\%), and CD133Low/ALDH1-High (1.1\%). The correlation between CD133/ALDH1 phenotypes and clinicopathological characteristics of CRC patients was evaluated using the one-way ANOVA and Tukey's post hoc analysis (Table 3). A marked positive correlation was only observed between the CD133-High/ALDH1-Low phenotype and neural invasion representing that this phenotype was more dominant in $\mathrm{CRC}$ patients compared to other phenotypes $(P=0.037)$.

Table 3. Correlation between CD133/ALDH1 phenotypes and clinicopathological characteristics in CRC.

\begin{tabular}{|c|c|c|c|c|c|}
\hline \multirow{2}{*}{$\begin{array}{l}\text { Patients and tumor } \\
\text { characteristics }\end{array}$} & \multicolumn{4}{|c|}{ CD133/ALDH1 phenotypes } & \multirow{2}{*}{$\boldsymbol{P}$} \\
\hline & $\begin{array}{l}\text { CD133High/ } \\
\text { ALDH1High }\end{array}$ & $\begin{array}{c}\text { CD133Low/ALDH } \\
\text { 1Low }\end{array}$ & $\begin{array}{l}\text { CD133High/ } \\
\text { ALDH1Low }\end{array}$ & $\begin{array}{l}\text { CD133Low/ } \\
\text { ALDH1High }\end{array}$ & \\
\hline \multicolumn{6}{|l|}{ Gender } \\
\hline Male & $13(8.3)$ & $17(10.9)$ & $125(80.1)$ & $1(0.6)$ & 0.49 \\
\hline Female & $12(9.4)$ & $8(6.3)$ & $105(82.7)$ & $2(1.6)$ & \\
\hline \multicolumn{6}{|l|}{ Age } \\
\hline$\leq 60$ & $14(9.4)$ & $13(8.7)$ & $121(81.2)$ & $1(0.7)$ & 0.90 \\
\hline$>60$ & $11(8.2)$ & $12(9)$ & $109(81.3)$ & $2(1.5)$ & \\
\hline \multicolumn{6}{|l|}{ Tumor size } \\
\hline$\leq 5$ & $14(7.3)$ & $16(8.3)$ & $160(82.9)$ & $3(1.6)$ & 0.32 \\
\hline$>5$ & $11(12.2)$ & $9(10)$ & $70(77.8)$ & $0(0)$ & \\
\hline \multicolumn{6}{|l|}{ Vascular invasion } \\
\hline Yes & $4(12.1)$ & $2(6.1)$ & $27(81.8)$ & $0(0)$ & 0.76 \\
\hline No & $21(8.4)$ & $23(9.2)$ & $203(81.2)$ & $3(1.2)$ & \\
\hline \multicolumn{6}{|l|}{ Neural invasion } \\
\hline Yes & $0(0)$ & $1(2.3)$ & $42(95.5)$ & $1(2.3)$ & 0.03 \\
\hline No & $25(10.5)$ & $24(10.1)$ & $188(78.6)$ & $2(0.8)$ & \\
\hline \multicolumn{6}{|c|}{ Lymph node involvement } \\
\hline Yes & $9(9.4)$ & $7(7.3)$ & $80(83.3)$ & $0(0)$ & 0.56 \\
\hline No & $16(8.6)$ & $18(9.6)$ & $150(80.2)$ & $3(1.6)$ & \\
\hline \multicolumn{6}{|l|}{ Metastasis } \\
\hline Yes & $1(5.6)$ & $2(11.1)$ & $15(83.3)$ & $0(0)$ & 0.90 \\
\hline No & $24(9.1)$ & $23(8.7)$ & $215(81.1)$ & $3(1.1)$ & \\
\hline \multicolumn{6}{|l|}{ Differentiation } \\
\hline Well & $11(10.2)$ & $9(8.3)$ & $85(78.7)$ & $3(2.8)$ & 0.14 \\
\hline Moderate \& Poor & $14(8)$ & $16(9.1)$ & $145(82.9)$ & $0(0)$ & \\
\hline
\end{tabular}

*The values shown in bold are statistically significant

\section{Discussion}

Colorectal cancer is one of the commonest visceral cancers and a leading cause of death throughout the world (27). Despite the improving trend of diagnostic and therapeutic processes, the majority of CRC patients experience a poor prognostic disease which is often manifested by drug resistance, recurrence, and metastasis (30). It is believed that cancers may be gradually organized by their own CSCs as a rare and small sub-population of cancer cells with potential to cause metastasis and recurrence (42-45). Several studies have been performed in this regard to identify specific markers for CRC CSCs but there is still controversy over the special marker to distinguish a distinct CSC population (12, 18, 32, 46). Therefore, discovery of more precise CSC markers would be helpful in early diagnosis, prognostic classification and well-organized targeted therapy of CRC and for improving prognosis through the metastasis and local recurrence prevention. The current study was carried out to assess the expression pattern of CD133 and ALDH1 as putative cancer stem cell markers in CRC using TMA and IHC techniques among Iranian population for the first time. We showed the increased expression of CD133 in the majority of CRC tumor samples compared to ALDH1. Lymph node, vascular, and neural invasion were more common in cases with higher CD133 expression than in cases with negative or low CD133 expression, which were statistically significant $(\mathrm{P}<0.05)$. In line with our findings, a metaanalysis based on the 37 studies reported increased CD133 expression in CRC as a poor prognostic factor in CRC patients and it was positively correlated with lymphatic and vascular invasion, distant metastasis, and tumor $\mathrm{T}$ category (47). $\mathrm{Li}$ and colleagues demonstrated that an increased percentage of CD133 positive tumor cells was linked with poor prognosis in CRC patients with higher stage (48). We observed CD133 overexpression in CRC cases with advanced 
stages (III \& IV) but differences were not statistically significant $(P>0.05)$. In contrast, another study reported the correlation between early stage (stage I) and worse outcome in CRC patients as a robust predictor factor (49).

Regarding CD133 expression patterns, immunohistochemical technique has been applied in various studies to show that the CD133 was mainly expressed on luminal surface and cell membrane of tumors (47-54), while other studies detected that it could be expressed both on cytoplasm and membrane of tumor cells $(44,55)$. Differences in the clinicopathological significance of tumor stem cell markers including CD133 is largely affected by the expression patterns of these markers. Epithelial to mesenchymal transition (EMT) and finally an invasive and metastatic phenotype is considerably linked to the cytoplasmic to membranous shift in CD133 localization (49). The CD133 expression at luminal surface of CRC tumor glands has been reported as an independent predictive marker of CRC (55). Our IHC findings revealed that the CD133 was mainly located at the luminal surface of most tumor samples $(66.7 \%$ as $\mathrm{H}$-score and $75.9 \%$ as intensity of staining) which were significantly correlated with CRC neural invasion $(P<0.05)$. Kojima et al. reported the luminal and cytoplasmic expression of CD133 in colorectal cancer mainly in well and moderately differentiated tumors but not in the poorly differentiated form which is significantly correlated with distant metastasis (56). We observed the expression of CD133 not only in well and moderately differentiated tumors but also in poorly differentiated cells that is correlated with metastasis $(P<0.05)$. These results are consistent with Horst et al.'s study, who found CD133 expression at the luminal surface of CRC gland with lumina shedding as a predictive marker of poor prognosis (57).

ALDH1 is a kind of detoxifying enzyme and potential CSC markers have been recognized in different cancers including head and neck (30) and breast (58) and several studies has focused on its prognostic significance in different cancers (30). An increase in cell proliferation and invasion capacity has been attributed to the role of ALDH1 in CSC characteristics and in the biological features of tumors $(58,59)$. Zhou and colleagues have demonstrated the relation between high expression of ALDH1 and poor outcome in CRC patients but it was not correlated to Lymph node invasion compared to CD133 (59). Lugli et al. reported no correlation between the ALDH1 expression and survival (59). They detected high ALDH1 expression in only $23.3 \%$ of CRC cases without any differences in survival rate (30). Although a large number of studies have investigated the role of ALDH1 expression on CRC patient's outcomes, controversy regarding prognostic significance of ALDH1 still remains. Therefore, further research should be conducted to reach a definite conclusion. Our study indicated that the ALDH1 CSC marker was only overexpressed in $40 \%$ of CRC patients which are partly consistent with the findings of the Lugli study (60). Further significant findings of the present study was that well and moderately differentiated tumors had smaller tumor size and exhibited a lower expression of ALDH1 in terms of $\mathrm{H}$-score compared with poorly differentiated tumors which was statistically significant $(P=0.001)$. Meanwhile, a meta-analysis study on the prognostic value of ALDH1, as a cancer stem cell marker indicated that ALDH1 was expressed in different levels among various populations of CRC patients (18). Nevertheless, differences between expression degrees of CSC markers and their complicated mechanisms are not yet clear. Liu et al. (2014) supposed that there were differences in expression patterns of ALDH1 and its prognostic significance between western and eastern people (61). Chen and colleagues systematically reviewed a large population of patients from western and eastern countries and concluded that the expression rate of ALDH1 is higher among the western populations (52\%) compared to low rate expression among the eastern populations (39\%).

Since CRC cells expressing one of putative CSC markers displayed high tumorigenicity especially in combination with other CSC markers (62), the assessment of combined markers may be helpful in better understanding tumor characteristics than those considered individually. Co-expression of CD133 and ALDH1 has been seen in a wide range of tumor cells, including lung (61) and gastric cancers which are involved in tumor invasion, metastasis and poor prognosis of patients (62). Therefore, we assessed the prognostic significance of combined CD133/ALDH1 phenotypes with clinicopathological factors in CRC. Our analysis revealed that CD133High/ALDH1Low phenotype was more frequent in CRC tumors than other combined phenotypes. There was also a positive significant correlation between this phenotype with neural invasion of tumors. Considering the different degree of CSC markers expression in several malignancies and differences among the various populations and markers localization, it can be concluded that CD133High/ALDH1Low phenotype may confer tumor progression behavior in CRC patients.

\section{Conclusion}

Combined detection of CD133 and ALDH1, as CSC associated markers, is likely to be valuable in understanding their clinicopathological and prognostic significance in CRC. Furthermore, patient classification in combined expression phenotypes of putative CSC markers could be identified as appropriate targeted therapies according to different subgroups of CRC patients and thus management of tumor progression, especially in advanced cases.

\section{Acknowledgements}

This study was supported by Iran University of Medical Sciences (Grant\#28786).

\section{Conflict of Interest}

The authors confirm that there are no known conflicts of interest associated with this publication, 
and there has been no significant financial support for this work that could have influenced its outcome.

\section{References}

1. Sadek SA, DM AR, Fatima S. The role of tumor budding in colorectal adenocarcinoma: Possible involvement of the intestinal cancer stem cell marker Lgr5. Indian J Pathol Microbiol. 2020;63(1):32-7. [DOI:10.4103/IJPM.IJPM 154 19] [PMID]

2. Siegel R, DeSantis C, Jemal A. Colorectal cancer statistics, 2014. CA Cancer J. Clin. 2014;64(2):104-17. [DOI:10.3322/caac.21220] [PMID]

3. Stewart BW, Kleihues P. World cancer report. 2003.

4. Mármol I, Sánchez-de-Diego C, Pradilla Dieste A, Cerrada E, Rodriguez Yoldi MJ. Colorectal carcinoma: a general overview and future perspectives in colorectal cancer. Int J Mol Sci. 2017;18(1):197. [DOI:10.3390/ijms18010197] [PMID] [PMCID]

5. Fearon ER, Vogelstein B. A genetic model for colorectal tumorigenesis. Cell. 1990;61(5):759-67. [DOI:10.1016/0092-8674(90)90186-I]

6. Ludwig JA, Weinstein JN. Biomarkers in cancer staging, prognosis and treatment selection. Nat Rev Cancer. 2005;5(11):845-56. [DOI:10.1038/nrc1739] [PMID]

7. Atreya CE, Sangale Z, Xu N, Matli MR, Tikishvili E, Welbourn $\mathrm{W}$, et al. PTEN expression is consistent in colorectal cancer primaries and metastases and associates with patient survival. Cancer medicine. 2013;2(4):496-506. [DOI:10.1002/cam4.97] [PMID] [PMCID]

8. Herzig DO, Tsikitis VL. Molecular markers for colon diagnosis, prognosis and targeted therapy. J Surg Oncol. 2015;111(1):96-102. [DOI:10.1002/jso.23806] [PMID]

9. Rosty C, Young JP, Walsh MD, Clendenning M, Sanderson K, Walters RJ, et al. PIK3CA activating mutation in colorectal carcinoma: associations with molecular features and survival. PLoS One. 2013;8(6):e65479.

[DOI:10.1371/journal.pone.0065479]

[PMID] [PMCID]

10. Sarli L, Bottarelli L, Bader G, Iusco D, Pizzi S, Costi R, et al. Association between recurrence of sporadic colorectal cancer, high level of microsatellite instability, and loss of heterozygosity at chromosome 18q. Dis Colon Rectum. 2004;47(9):1467-82. [DOI:10.1007/s10350-004-0628-6] [PMID]

11. Vaz MA, Martinez JC, Devesa JM, Trill JD, Abraira V, Riquelme A, et al. Prognostic value of stem cell quantification in stage II colon cancer. PLoS One. 2014;9(2):e88480.

[DOI:10.1371/journal.pone.0088480] [PMID] [PMCID]

12. Huang R, Mo D, Wu J, Ai H, Lu Y. CD133 expression correlates with clinicopathologic features and poor prognosis of colorectal cancer patients: An updated meta-analysis of 37 studies. Medicine (Baltimore). 2018;97(23):e10446.

[DOI:10.1097/MD.0000000000010446] [PMID] [PMCID]

13. Vermeulen L, de Sousa e Melo F, Richel DJ, Medema JP. The developing cancer stem-cell model: clinical challenges and opportunities. Lancet Oncol. 2012;13(2):e83-9.

2045(11)70257-1]

[DOI:10.1016/S1470-

14. Boman B, Wicha M. Cancer Stem Cells: A Step toward the Cure. American Society of Clinical Oncology. 2008; 26 (17): 2795-2799. [DOI:10.1200/JCO.2008.17.7436] [PMID]

15. Dick JE. Looking ahead in cancer stem cell research. Nat Biotechnol. 2009;27(1):44-6. [DOI:10.1038/nbt0109-44] [PMID]

16. Zhou J-Y, Chen M, Ma L, Wang X, Chen Y-G, Liu SL. Role of CD44high/CD133high HCT-116 cells in the tumorigenesis of colon cancer. Oncotarget. 2016;7(7):7657. [DOI:10.18632/oncotarget.7084] [PMID] [PMCID]

17. Leng Z, Xia Q, Chen J, Li Y, Xu J, Zhao E, et al. Lgr5+CD44+EpCAM+ Strictly Defines Cancer Stem Cells in Human Colorectal Cancer. Cell Physiol Biochem. 2018;46(2):860-72. [DOI:10.1159/000488743] [PMID]

18. Cherciu I, Barbalan A, Pirici D, Margaritescu C, Saftoiu A. Stem cells, colorectal cancer and cancer stem cell markers correlations. Curr Health Sci J. 2014;40(3):153-61.

19. Vaiopoulos AG, Kostakis ID, Koutsilieris M, Papavassiliou AG. Colorectal cancer stem cells. Stem Cells. 2012;30(3):363-71. [DOI:10.1002/stem.1031] [PMID]

20. Thenappan A, Li Y, Shetty K, Johnson L, Reddy EP, Mishra L. New Therapeutics Targeting Colon Cancer Stem Cells. Curr Colorectal Cancer Rep. 2009;5(4):209. [DOI:10.1007/s11888-009-0029-2] [PMID] [PMCID]

21. O'Brien CA, Pollett A, Gallinger S, Dick JE. A human colon cancer cell capable of initiating tumour growth in immunodeficient mice. Nature. 2007;445(7123):10610. [DOI:10.1038/nature05372] [PMID]

22. Miraglia S, Godfrey W, Yin AH, Atkins K, Warnke R, Holden JT, et al. A novel five-transmembrane hematopoietic stem cell antigen: isolation, characterization, and molecular cloning. Blood. 1997;90(12):5013-21.

[DOI:10.1182/blood.V90.12.5013] [PMID]

23. Corbeil D, Roper K, Fargeas CA, Joester A, Huttner WB. Prominin: a story of cholesterol, plasma membrane protrusions and human pathology. Traffic. 2001;2(2):82-91. 0854.2001.020202.x] [PMID]

24. Cheng J-X, Liu B-L, Zhang X. How powerful is CD133 as a cancer stem cell marker in brain tumors? Cancer Treat Rev. 2009;35(5):403-8. [DOI:10.1016/j.ctrv.2009.03.002] [PMID]

25. Richardson GD, Robson CN, Lang SH, Neal DE, Maitland NJ, Collins AT. CD133, a novel marker for human prostatic epithelial stem cells. J Cell Sci. 2004;117(Pt 16):3539-45. [DOI:10.1242/jcs.01222] [PMID]

26. Vander Griend DJ, Karthaus WL, Dalrymple S, Meeker A, DeMarzo AM, Isaacs JT. The role of CD133 in normal human prostate stem cells and malignant cancerinitiating cells. Cancer Res. 2008;68(23):9703-11. [DOI:10.1158/0008-5472.CAN-08-3084] [PMID] [PMCID] 
27. Wang K, Xu J, Zhang J, Huang J. Prognostic role of CD133 expression in colorectal cancer: a meta-analysis. BMC Cancer. 2012;12(1):573. [DOI:10.1186/14712407-12-573] [PMID] [PMCID]

28. Ricci-Vitiani L, Lombardi DG, Pilozzi E, Biffoni M, Todaro M, Peschle C, et al. Identification and expansion of human colon-cancer-initiating cells. Nature. 2007;445(7123):111-5. [DOI:10.1038/nature05384] [PMID]

29. Ieta K, Tanaka F, Haraguchi N, Kita Y, Sakashita H, Mimori K, et al. Biological and genetic characteristics of tumor-initiating cells in colon cancer. Ann Surg Oncol. 2008;15(2):638-48. [DOI:10.1245/s10434-0079605-3] [PMID]

30. Galizia G, Gemei M, Del Vecchio L, Zamboli A, Di Noto R, Mirabelli P, et al. Combined CD133/CD44 expression as a prognostic indicator of disease-free survival in patients with colorectal cancer. AMA Arch Surg. [DOI:10.1001/archsurg.2011.795] [PMID]

31. Chen J, Xia Q, Jiang B, Chang W, Yuan W, Ma Z, et al. Prognostic Value of Cancer Stem Cell Marker ALDH1 Expression in Colorectal Cancer: A Systematic Review and Meta-Analysis. PLoS One. 2015;10(12):e0145164. [DOI:10.1371/journal.pone.0145164] [PMID] [PMCID]

32. Vishnubalaji R, Manikandan M, Fahad M, Hamam R, Alfayez M, Kassem M, et al. Molecular profiling of ALDH1(+) colorectal cancer stem cells reveals preferential activation of MAPK, FAK, and oxidative stress pro-survival signalling pathways. Oncotarget. 2018;9(17):13551-64.

[DOI:10.18632/oncotarget.24420] [PMID] [PMCID]

33. Kozovska Z, Patsalias A, Bajzik V, Durinikova E, Demkova L, Jargasova S, et al. ALDH1A inhibition sensitizes colon cancer cells to chemotherapy. BMC Cancer. 2018;18(1):656. [DOI:10.1186/s12885-0184572-6] [PMID] [PMCID]

34. Tanei T, Morimoto K, Shimazu K, Kim SJ, Tanji Y, Taguchi T, et al. Association of breast cancer stem cells identified by aldehyde dehydrogenase 1 expression with resistance to sequential Paclitaxel and epirubicin-based chemotherapy for breast cancers. Clin Cancer Res. 2009;15(12):4234-41. [DOI:10.1158/1078-0432.CCR08-1479] [PMID]

35. Rasheed ZA, Yang J, Wang Q, Kowalski J, Freed I, Murter $\mathrm{C}$, et al. Prognostic significance of tumorigenic cells with mesenchymal features in pancreatic adenocarcinoma. J Natl Cancer Inst. 2010;102(5):34051. [DOI:10.1093/jnci/djp535] [PMID] [PMCID]

36. Penumatsa K, Edassery SL, Barua A, Bradaric MJ, Luborsky JL. Differential expression of aldehyde dehydrogenase 1al (ALDH1) in normal ovary and serous ovarian tumors. J Ovarian Res. 2010;3(1):28. [DOI:10.1186/1757-2215-3-28] [PMID] [PMCID]

37. Serrano D, Bleau AM, Fernandez-Garcia I, FernandezMarcelo $\mathrm{T}$, Iniesta $\mathrm{P}$, Ortiz-de-Solorzano $\mathrm{C}$, et al. Inhibition of telomerase activity preferentially targets aldehyde dehydrogenase-positive cancer stem-like cells in lung cancer. Mol Cancer. 2011;10(1):96. [DOI:10.1186/1476-4598-10-96] [PMID] [PMCID]

38. Moreb J, Zucali J, Zhang Y, Colvin M, Gross M. Role of aldehyde dehydrogenase in the protection of hematopoietic progenitor cells from 4- hydroperoxycyclophosphamide by interleukin $1 \beta$ and tumor necrosis factor. Cancer Res. 1992;52(7):1770-4.

39. Ginestier C, Hur MH, Charafe-Jauffret E, Monville F, Dutcher J, Brown M, et al. ALDH1 is a marker of normal and malignant human mammary stem cells and a predictor of poor clinical outcome. Cell stem cell. 2007;1(5):555-67. [DOI:10.1016/j.stem.2007.08.014] [PMID] [PMCID]

40. Huang EH, Hynes MJ, Zhang T, Ginestier C, Dontu G, Appelman H, et al. Aldehyde dehydrogenase 1 is a marker for normal and malignant human colonic stem cells (SC) and tracks SC overpopulation during colon tumorigenesis. Cancer Res. 2009;69(8):3382-9. [DOI:10.1158/0008-5472.CAN-08-4418] [PMID] [PMCID]

41. Deng Y, Zhou J, Fang L, Cai Y, Ke J, Xie X, et al. ALDH1 is an independent prognostic factor for patients with stages II-III rectal cancer after receiving radiochemotherapy. Br J Cancer. 2014;110(2):430-4. [DOI:10.1038/bjc.2013.767] [PMID] [PMCID]

42. O'Dwyer D, Ralton LD, O'Shea A, Murray GI. The proteomics of colorectal cancer: identification of a protein signature associated with prognosis. PLoS One. 2011;6(11):e27718.

[DOI:10.1371/journal.pone.0027718] [PMCID]

[PMID]

43. Goossens-Beumer IJ, Zeestraten EC, Benard A, Christen T, Reimers MS, Keijzer R, et al. Clinical prognostic value of combined analysis of Aldh1, Survivin, and EpCAM expression in colorectal cancer. $\mathrm{Br} \quad \mathrm{J} \quad$ Cancer. 2014;110(12):2935-44. [DOI:10.1038/bjc.2014.226] [PMID] [PMCID]

44. Sedaghat S, Gheytanchi E, Asgari M, Roudi R, Keymoosi H, Madjd Z. Expression of Cancer Stem Cell Markers OCT4 and CD133 in Transitional Cell Carcinomas. Appl Immunohistochem Mol Morphol. 2017;25(3):196-202.

[DOI:10.1097/PAI.0000000000000291] [PMID]

45. Camp RL, Charette LA, Rimm DL. Validation of tissue microarray technology in breast carcinoma. Lab Invest. 2000;80(12):1943-9. [DOI:10.1038/labinvest.3780204] [PMID]

46. Clevers H. The cancer stem cell: premises, promises and challenges. Nat Med. 2011;17(3):313-9. [DOI:10.1038/nm.2304] [PMID]

47. Rich JN. Cancer stem cells in radiation resistance. Cancer Res. 2007;67(19):8980-4. [DOI:10.1158/00085472.CAN-07-0895] [PMID]

48. Chen S, Song X, Chen Z, Li X, Li M, Liu H, et al. CD133 expression and the prognosis of colorectal cancer: a systematic review and meta-analysis. PLoS One. 2013;8(2):e56380. [DOI:10.1371/journal.pone.0056380] [PMID] [PMCID]

49. He S, Zhou H, Zhu X, Hu S, Fei M, Wan D, et al. Expression of Lgr5, a marker of intestinal stem cells, in colorectal cancer and its clinicopathological significance. Biomed Pharmacother. 2014;68(5):50713. [DOI:10.1016/j.biopha.2014.03.016] [PMID]

50. Li C-Y, Li B-X, Liang Y, Peng R-Q, Ding Y, Xu D-Z, et al. Higher percentage of CD133+ cells is associated with poor prognosis in colon carcinoma patients with stage IIIB. J Transl Med. 2009;7(1):56. [DOI:10.1186/1479-5876-7-56] [PMID] [PMCID] 
51. Reggiani Bonetti L, Migaldi M, Caredda E, Boninsegna A, Ponz De Leon M, Di Gregorio C, et al. Increased expression of CD133 is a strong predictor of poor outcome in stage I colorectal cancer patients. Scand J Gastroenterol. 2012;47(10):1211-7. [DOI: 10.3109/00365521.2012.694904] [PMID]

52. Horst D, Kriegl L, Engel J, Kirchner T, Jung A. Prognostic significance of the cancer stem cell markers CD133, CD44, and CD166 in colorectal cancer. Cancer Invest. [DOI:10.1080/07357900902744502] [PMID]

53. Kojima M, Ishii G, Atsumi N, Fujii S, Saito N, Ochiai A. Immunohistochemical detection of CD133 expression in colorectal cancer: a clinicopathological study. Cancer Sci. 2008;99(8):1578-83. [DOI:10.1111/j.1349-7006.2008.00849.x] [PMID]

54. Wang Q, Chen ZG, Du CZ, Wang HW, Yan L, Gu J. Cancer stem cell marker CD133+ tumour cells and clinical outcome in rectal cancer. Histopathology. 2009;55(3):284-93. [DOI:10.1111/j.13652559.2009.03378.x ] [PMID]

55. Kojima M, Ishii G, Atsumi N, Nishizawa Y, Saito N, Ochiai A. CD133 expression in rectal cancer after preoperative chemoradiotherapy. Cancer Sci. 2010;101(4):906-12. [DOI:10.1111/j.13497006.2009.01478.x ] [PMID]

56. García VM, Batlle J, Casado E, Burgos E, de Castro J, Belda $\mathrm{C}$, et al. Immunohistochemical analysis of tumour regression grade for rectal cancer after neoadjuvant chemoradiotherapy. Colorectal Dis. 2011;13(9):989-98. [DOI:10.1111/j.1463-1318.2010.02386.x] [PMID]

57. Nagata T, Sakakura C, Komiyama S, Miyashita A, Nishio M, Murayama Y, et al. Expression of cancer stem cell markers CD133 and CD44 in locoregional recurrence of rectal cancer. Anticancer Res. 2011;31(2):495-500.

58. Coco C, Zannoni GF, Caredda E, Sioletic S, Boninsegna A, Migaldi M, et al. Increased expression of CD133 and reduced dystroglycan expression are strong predictors of poor outcome in colon cancer patients. J Exp Clin Cancer Res. 2012;31(1):71. [DOI:10.1186/1756-996631-71] [PMID] [PMCID]

59. Li H, Zhao P, Lu Y, Lu Y. Correlation of aberrant expression of CD133 with FHIT and malignant phenotype of colorectal adenocarcinoma. Int $\mathrm{J}$ Colorectal Dis. 2012;27(8):1015-20. [DOI:10.1007/s00384-012-1421-y] [PMID]
60. Horst D, Kriegl L, Engel J, Kirchner T, Jung A. CD133 expression is an independent prognostic marker for low survival in colorectal cancer. $\mathrm{Br} \mathrm{J}$ Cancer. 2008;99(8):1285-9. [DOI:10.1038/sj.bjc.6604664] [PMID] [PMCID]

61. Zhou C, Sun B. The prognostic role of the cancer stem cell marker aldehyde dehydrogenase 1 in head and neck squamous cell carcinomas: a meta-analysis. Oral Oncol. 2014;50(12):1144-8. [DOI:10.1016/j.oraloncology.2014.08.018] [PMID]

62. Korfias D, Contis J, Frangou-Plemenou M, Gennatas K, Kondis A, Vlachodimitropoulos D. Stem cells in ductal breast cancer: immunohistochemical expression of CD44, CD24, CD133, and ALDH-1 markers in 104 cases. Eur J Gynaecol Oncol. 2020;41(1):36-41. [DOI:10.31083/j.ejgo.2020.01.4810]

63. Zhou F, Mu YD, Liang J, Liu ZX, Chen HS, Zhang JF. Expression and prognostic value of tumor stem cell markers ALDH1 and CD133 in colorectal carcinoma. Oncol Lett. 2014;7(2):507-12. [DOI:10.3892/ol.2013.1723] [PMID] [PMCID]

64. Lugli A, Iezzi G, Hostettler I, Muraro MG, Mele V, Tornillo L, et al. Prognostic impact of the expression of putative cancer stem cell markers CD133, CD166, CD44s, EpCAM, and ALDH1 in colorectal cancer. Br J Cancer. 2010;103(3):382-90. [DOI:10.1038/sj.bjc.6605762] [PMID] [PMCID]

65. Liu Y, Lv DL, Duan JJ, Xu SL, Zhang JF, Yang XJ, et al. ALDH1A1 expression correlates with clinicopathologic features and poor prognosis of breast cancer patients: a systematic review and meta-analysis. BMC Cancer. 2014;14(1):444. [DOI:10.1186/14712407-14-444] [PMID] [PMCID]

66. Koren A, Rijavec M, Kern I, Sodja E, Korosec P, Cufer T. BMI1, ALDH1A1, and CD133 Transcripts Connect Epithelial-Mesenchymal Transition to Cancer Stem Cells in Lung Carcinoma. Stem Cells Int. 2016;2016:9714315. [DOI:10.1155/2016/9714315] [PMID] [PMCID]

67. Liu WT, Liu WB, Gao M, Zhang YY, Gu KS. Expression of ALDH1A1 and CD133 is associated with the prognosis and effect of different chemotherapeutic regimens in gastric cancer. Oncol Lett. 2019;18(5):4573-82. [DOI:10.3892/ol.2019.10798] [PMID] [PMCID]

\section{How to Cite This Article}

Rezaee, M., Gheytanchi, E., Madjd, Z., Mehrazma, M. Clinicopathological Significance of Tumor Stem Cell Markers ALDH1 and CD133 in Colorectal Carcinoma. Iranian Journal of Pathology, 2020; 16(1): 40-50. doi: 10.30699/ijp.2020.127441.238916 